\title{
Mycorrhiza-like Structures in Rooted Microshoots of Pinus pinea L.
}

\author{
M.R. Castro, a, , C. Ragonezi ${ }^{1}$, K. Klimaszewska ${ }^{2}$, M. Lima ${ }^{1}$, P. de Oliveira ${ }^{1}$ and \\ M.A. Zavattieri ${ }^{1}$ \\ ${ }^{1}$ Laboratory of Plant Breeding and Biotechnology, ICAM, University of Évora, Apart. 94, \\ 7002-554 Evora, Portugal \\ ${ }^{2}$ Natural Resources Canada, Canadian Forest Service, Laurentian Forestry Centre, 1055 \\ du P.E.P.S. PO Box 10380, Stn.Sainte-Foy, Québec, G1V 4C7, Canada
}

Keywords: ectomycorrhizae, stone pine, rhizotron, adventitious roots, acclimation, biotization

\begin{abstract}
Pinus pinea $\mathrm{L}$. (stone pine) is one of the major plantation species in Iberian Peninsula, being Portugal the largest edible seed producer in the world. The induction and improvement of in vitro rhizogenesis of microshoots of Pinus pinea was developed in our laboratory using a co-culture system with ECM fungi. In the acclimation phase in mixed substrates, or in rhizotrons, anatomical and morphological studies were done to observe the evolution of the root system in microshoots from the co-culture system vs. control plants. Extensive dichotomous and coralloid branching of lateral roots occurred spontaneously in inoculated and control plants as well. Moreover, similar branching occurred in liquid culture of excised seedling roots without the presence of ECM fungi. The striking similarity of these organs with pine ectomycorrhizas prompted their anatomical analysis; however the presence of Hartig net was not confirmed. These results suggested that the development of ECM-like structures might have occurred spontaneously.
\end{abstract}

\section{INTRODUCTION}

Mycorrhizas are symbiotic structures formed between plant roots and fungi that act as an extension of absorption system, where the fungal partner obtains photosynthetic sugars from the host plant while, in return, the plant receives mineral nutrients from the fungus (Smith and Read, 1997). Ectomycorrhizas (ECM) are the main absorption organs in conifers, and the exchange of nutrients occurs in a specific structure that is formed between the fungal hyphae and the outer root layers. This structure is known as the Hartig net, which is formed by the hyphae penetrating from the surrounding mantle into the root apoplast (Smith and Read, 1997; Brundrett et al., 1996). This mutualistic relationship with fungi grants conifers an ecological advantage to withstand the harsh living conditions.

Stone pine is an extremely appreciated edible nut producer and one of the major plantation species in the Iberian Peninsula. Achieving its clonal propagation is a major goal in the biotechnological development for this species, but has met overwhelming difficulties. The complex interactions between pines and their ECM partners suggest that they might be capable of overcoming such difficulties. The objective of our work was to ameliorate the in vitro adventitious rooting (which does not develop well in the agar cultures) by co-culturing with ECM fungi collected from Stone pine stands (Oliveira et al., 2003). It was also to characterize the fungi-root interactions that enable the development of roots in microshoots and to identify the signalling mediators between ectomycorrhizal fungi and stone pine roots. These potentially new insights into the interactions that take place in the pine rhizosphere could allow us to develop an axenic system that mimics the promoting effect of ECM fungi.

\footnotetext{
a marinho_dorito@yahoo.com
} 


\section{MATERIALS AND METHODS}

\section{Plant Material}

Mature seeds of stone pine were obtained in March 2007 from selected 'plus' trees (Alcácer do Sal Region, Portugal) and were stored in a cold chamber at $4^{\circ} \mathrm{C}$ until used.

\section{Shoot Organogenesis} et al. (2008).

For the description of shoot organogenesis, see Oliveira et al. (2003) and Ragonezi

\section{Root Organogenesis}

Microshoots where placed in rooting medium. The media used were WPMRI and WPMRE (woody plant medium root induction and expression, respectively) (Ragonezi et al., 2008).

\section{Fungi}

Fungi were isolated from single ectomycorrhizas as described by Oliveira et al. (2003) collected at the same location as the cones used for obtaining mature seeds for organogenesis experiments. The fungi were maintained in pure culture using standard procedures (Brundrett et al., 1996). Sixty-five independent isolates were obtained (unpublished results) and screened for their effect on root growth, many of which are included in the present study.

\section{In Vitro Co-Culture}

Following the rooting induction and expression phase, the individuals that showed root development were transferred to the double-layer medium and, after a brief period of adaptation to the medium, inoculated with selected fungi, with matching controls to monitor root growth in the absence of inoculation (Oliveira et al., 2003).

\section{Acclimation}

The plantlets were transferred to vermiculite for two weeks and then transferred two different substrate systems to observe the development of the root system: mixed substrate in containers, or peat in a rhizotron (Finlay and Read, 1986). During acclimation plants grew in a growth chamber at $25 / 19^{\circ} \mathrm{C}$ day/night temperatures, with $16 \mathrm{~h}$ photoperiod $\left(270 \mu \mathrm{mol} \mathrm{s}^{-1} \mathrm{~m}^{-2}\right)$ for 10 weeks. The relative humidity of the growth chamber started at $80 \%$ and gradually decreased to $60 \%$. Plants were watered as required with alternating sterile water and liquid WPM (macronutrients only).

\section{Rhizotron}

Rhizotrons allow the visualization of root development whenever desired without disturbing the normal functions of the plants. Basically, they are made of two acrylic plates, $20 \times 20 \mathrm{~cm}$ each, with interval made by $5 \mathrm{~mm}$ spacers and filled with turf, in order to support and feed the plants, as suggested by Finlay and Read (1986) with some adaptations from Bending and Read (1996). The root growth was observed thereafter for 4 to 6 weeks, the time required for most of the plants to explore the available space in the rhizotron. Rhizotrons were very useful for the identification of target structures, as well as other symbiotic features that could then be extracted for further studies with minimal disturbance to the rest of the root architecture.

\section{Axenic Root Cultures}

Root segments of $2 \mathrm{~cm}$ long obtained from germinated seeds were excised and were cultured in liquid medium in an orbital shaker $(125 \mathrm{rpm})$ according to Kaska et al. (1999) for 3 to 4 weeks. Afterward, the roots were photographed and used for histological comparison. 


\section{Root Microscopy}

Two different methods were used to study root anatomy: 1) hand sections of root segments were obtained according to Brundrett (2008): segments were placed between pieces of laboratory Parafilm ${ }^{\circledR}$ and cut as thin as possible using a razor blade under a Zoom Stereo Research Microscope 7-70X Olympus SZH10. Structural details of the root anatomy and in some cases of the Hartig-net of ECM (when found) were observed under Light Microscope with image acquisition device Olympus CX-40 and photographs were taken with a Canon Power-Shot A630 camera. 2) Dichotomous and short roots were fixed in FAA, dehydrated in an aqueous series of ethanol $(70,80,95,100 \%)$, clarified in xylol, embedded in paraffin and cut with a rotary microtome $(8-10 \mu \mathrm{m})$. The sections were stained with toluidine blue, mounted in entellan acid and observed under light microscope (Giomaro et al., 2000).

\section{RESULTS AND DISCUSSION}

Adventitious roots regenerated by stone pine microshoots as well as axenic embryo root cultures developed mycorrhizal-like (dichotomous) root laterals even without fungal infection. This is not exceptional and was observed for intact plants as well as for excised roots. In the former, these structures appeared in all experimental settings tested, with the frequency of dichotomous branching increasing as a consequence of a reduction of macro-nutrients in the medium (compared with complete macronutrients) and also in cultures that spent more than one month on the same co-culture medium. These results suggested that without any fungus interaction, some stone pine genotypes responded to less favourable in vitro growing conditions (i.e., lower nutrient concentration and/or lower water availability) by producing mycorrhizal coralloid-like structures. Also coralloid structures appeared as a consequence of applied naphthalene acetic acid (NAA) in the induction medium (Fig. 1b).

On the other hand, under in vitro co-culture (pine-fungus) there was a great variability in the plant response. Some combinations of pine clones with specific fungi showed dichotomous branching while others did not (Figs. 2a, b).

After a few months in substrate, at the moment of transferring to larger containers, dichotomous ectomycorrhizal-like root tips were detected for several inocula, each producing a different rootlet type, indicative of specificity in the development of these structures as a function of the provided inoculum. At least in these earlier stages, noninoculated controls were devoid of such structures (Fig. 3). Rhizotron-contained plants did not show any such developments. A careful examination of all ECM-like morphotypes failed to reveal Hartig net anatomical features, thus not confirming the symbiotic nature of these structures. The mycorrhizal potential of the fungal inocula is still under investigation, thus not allowing to determine whether that is due to the taxonomical identity of the fungi or the mimicry of dichotomous development by ECM fungi which might start a symbiotic relationship but failed to persist in that condition.

Some plants presented a great number of monopodial short roots with root hairs; others dichotomous branching without hairs and also dichotomy with hairs (Figs. 4a, b, c). Only through the histological observations it was possible to distinguish between true mycorrhizal symbioses and mycorrhizal-like structures as well as ectoendomycorrhizae, the latter not correlating with a particular co-culture inoculum, thence probably from contamination with E-type propagules (Figs. 5a, b).

Axenic root cultures showed profuse dichotomous branching similar to those of in vitro cultured plants (Fig. 6).

Many environmental or cultural conditions could have influenced the capacity of some stone pine clones to produce mycorrhizal-like structures. At the current stage of our study it is impossible to know if there is a correlation between pine clones and their propensity to form mycorrhizal-like structures. Responses to the inocula used in the coculture (either the resumed growth of roots previously formed as a consequence of plant growth regulators treatment, or dichotomous branching) was not consistently correlated with the induction of mycorrhizal symbiosis in the acclimation phase. Nevertheless, the 
characterization of biochemical signals that are likely mediating these effects will continue to be pursued, in order to understand the possible physiological implications for the plants from the development of these structures.

\section{CONCLUSIONS}

- This is the first report on the abundant mycorrhizal-like structures in stone pine roots that were produced by axenic cultures, in in vitro-cultures and in subsequent acclimation phase in mixed substrates.

- There was a strong similarity between extensive dichotomous and coralloid branching of lateral roots that grew spontaneously in stone pine with those derived from fungal inoculation.

- Due to this similarity it may be difficult to diagnose ectomycorrhizas without confirmation of the ECM status by histological analysis.

- Since this response appeared to be 'genotype dependent' more studies will be needed to establish correlation between stone pine clones and the root system morphology.

- The biochemical studies that are being carried out presently on the co-cultured roots could elucidate the nature of the compounds that cause a highly effective adventitious rooting in the presence of certain fungi and afterwards in the acclimation phase.

\section{ACKNOWLEDGEMENTS}

This work was supported by FCT Portugal: PTDC/AGR-CFL/71437/2006 Analysis and Mastering of Root Growth Signalling by Ectomycorrhizal Fungi on Pinus pinea Microshoot Cultures.

\section{Literature Cited}

Bending, G.D. and Read, D.J. 1996. Effects of the soluble polyphenol tannic acid on the activities of ericoid and ectomycorrhizal fungi. Soil Biology and Biochemistry 28:1595-1602.

Brundrett, M., Bougher, N., Dell, B., Grave, T. and Malajczuk, N. 1996. Isolation of fungi from within mycorrhizal roots. In: Working with Mycorrhizas in Forestry and Agriculture. Australian Centre for International Agricultural Research Monograph 32, Canberra, Australia, p.229.

Brundrett, M. 2008 http://mycorrhizas.info/download/pdf/4_10.pdf

Finlay, R.D. and Read, D.J 1986. The structure and function of the vegetative mycelium of ectomycorrhizal plants. I-Tranlocation of ${ }^{14} \mathrm{C}$-labeled carbon between plants interconnected by a common mycelium. New Phytologist 103:143-156.

Giomaro, G. et al. 2000. Anatomical and morphological characterization of mycorrhizas of five strains of Tuber borchii Vittad. Mycorrhiza 10:107-114.

Kaska, D.D. et al. 1999. Auxin transport inhibitors act through ethylene to regulate dichotomous branching of lateral root meristems in pine. New Phytologist 142:49-58.

Oliveira, P., Barriga, J., Cavaleiro, C., Peixe, A. and Potes, A.Z. 2003. Sustained in vitro root development obtained in Pinus pinea inoculated with ectomycorrhizal fungi. Forestry 76:579-587.

Ragonezi, C., Castro, M.R., Zavattieri, A. and Lima, M. Achieving in vitro rooting in recalcitrant pine. In: $5^{\text {th }}$ International Symposium on Adventitious Root Formation, Alcalá de Henares - Spain, June 2008.

Smith, S.E. and Read. D.J. 1997. Mycorrhizal Symbiosis, Second Edition. Academic Press. 


\section{Figures}
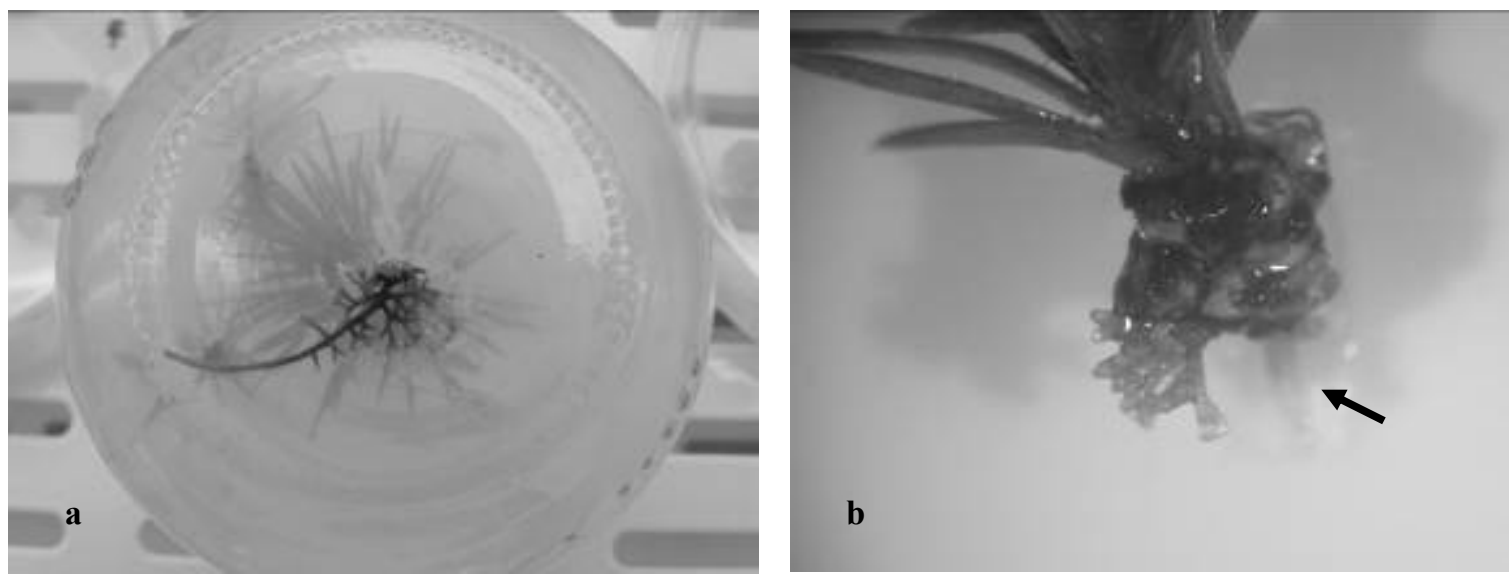

Fig. 1. (a) After more than one month without transferring to new medium, some genotypes developed coralloid mycorrhizal-like structures. (b) Coralloid structures also appeared as a consequence of NAA in the rooting induction medium. The arrow indicates a normal root type induced by auxin treatment.
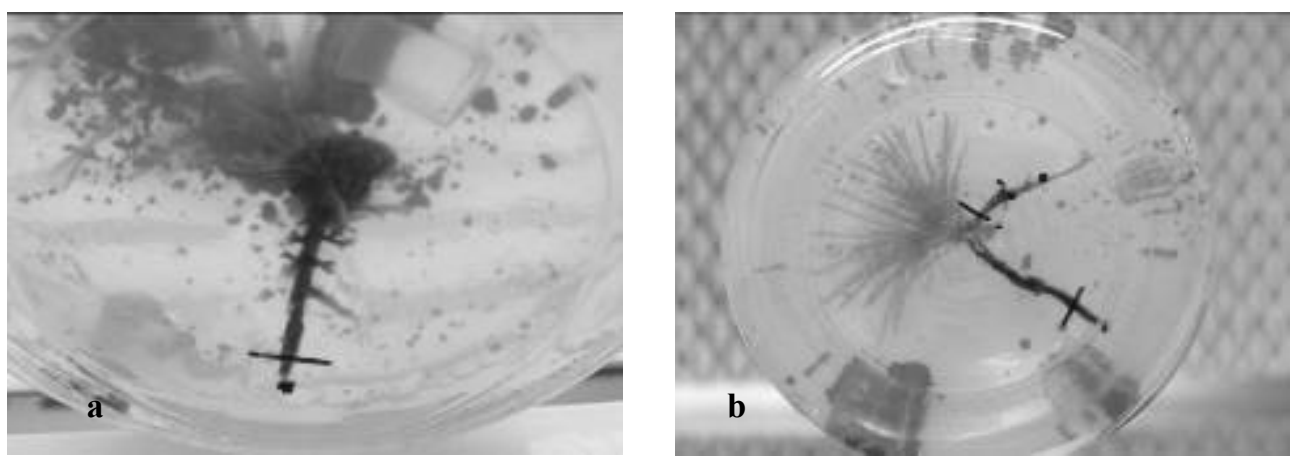

Fig. 2. (a) Differential responses in the in vitro adventitious root formation in co-culture system. Root with dichotomous branching. (b) Normal in vitro growth of the root system. Mycelium could be seen growing on the surface of the culture medium in both culture vessels. 

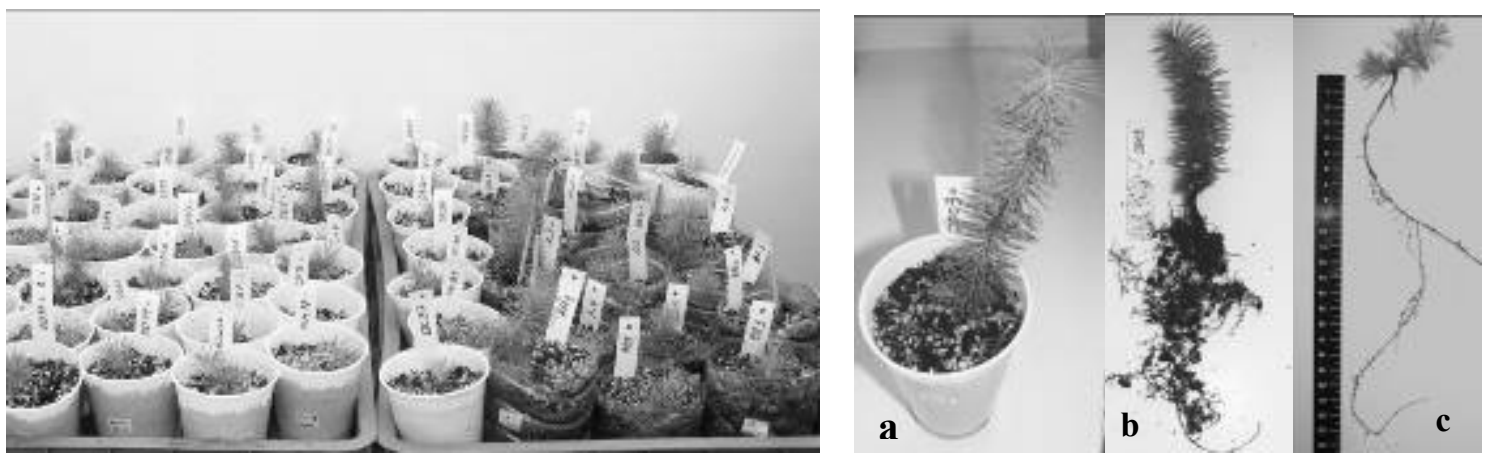

Fig. 3. Left: Rooted plants in the acclimation phase. Plants after 4 month in the acclimation phase. (a) Inoculated pine plant, identified as DD03, shows a good development of the aerial part and (b) also compact and dichotomous branched root system. (c) Control plant, not inoculated with less aerial part development and a linear root system $23 \mathrm{~cm}$ long.
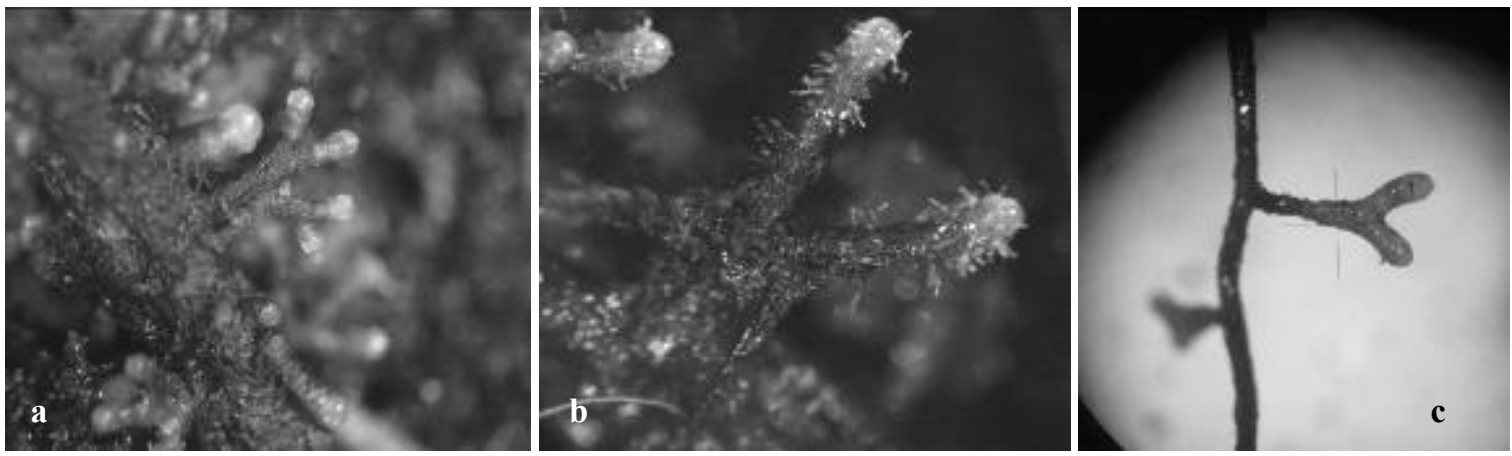

Fig. 4. (a) Different anatomical structures of the roots in plants derived from co-culture after 4 month in the acclimation phase (pine clones/different fungi isolates). Extensively dichotomous branching without hairs, (b) dichotomous short root with hairs and (c) ectomycorrhizal-like structure.
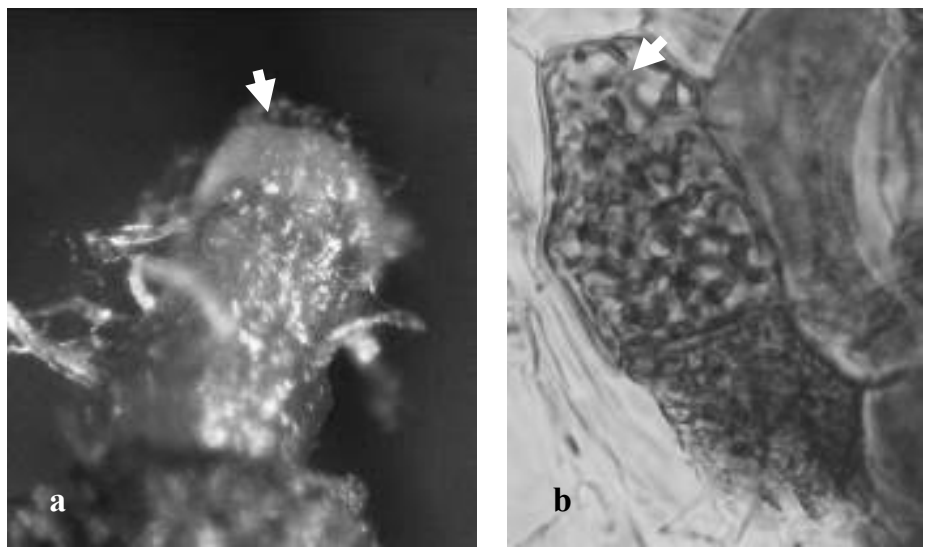

Fig. 5. (a) Short root covered with mycelia. (b) Longitudinal section shows the intracellular hyphae. 


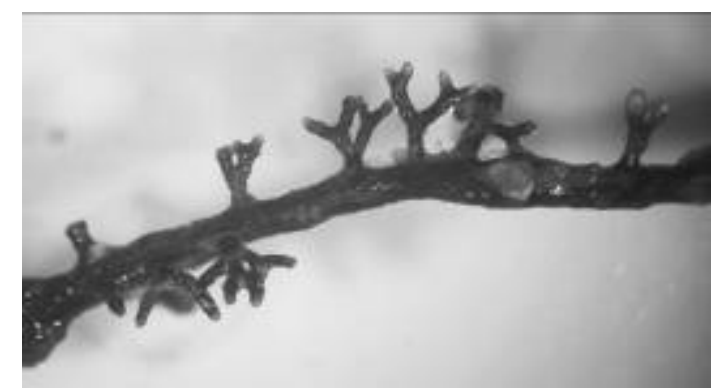

Fig. 6. Dichotomous branching observed in the liquid axenic root cultures after one month of growth. 
